\title{
Apports des techniques isotopiques à la connaissance des gisements d'eau minérale
}

\author{
B. Blavoux \\ Université d'Avignon
}

\section{I $\square$ INTRODUCTION}

Les sources minérales sont le produit de circuits hydrogéologiques longs, complexes et souvent profonds. La connaissance détaillée du circuit et de son fonctionnement est indispensable pour en assurer une gestion optimale. c'est-à-dire protéger le gisement, contrôler son exploitation comme éventuellement mobiliser une ressource supplémentaire qui puisse être authentifiée comme eau minérale.

Une bonne connaissance du circuit nécessite de répondre aux interrogations sur l'origine de l'eau et de sa minéralisation (où), sur l'histoire de l'eau depuis les parties profondes où elle a acquis l'essentiel de sa minéralisation jusqu'à la zone d'émergence (comment) et enfin sur la durée du circuit (quand).

Ce travail de recherche comporte donc pour l'essentiel :

- la localisation de la zone d'alimentation, du réservoir principal (nature des roches et profondeur), la caractéri- sation du drain de collecte et des roches rencontrées au cours de la remontée, la recherche de l'origine des gaz éventuels ;

- la connaissance des modifications de la composition chimique de l'eau lors des dernières étapes de son parcours souterrain, réactions chimiques et biochimiques, éventualité et ampleur d'un mélange avec des eaux plus superficielles :

- la détermination des vitesses et de l'âge de l'eau.

Les méthodes isotopiques se prêtent remarquablement à ce genre d'études et sont souvent performantes.

On utilise les rapports de deux isotopes stables d'un même composé (isotope lourd rare sur isotope léger banal), ceux de la molécule d'eau ${ }^{18} \mathrm{O} /{ }^{16} \mathrm{O}$ et ${ }^{2} \mathrm{H} /{ }^{1} \mathrm{H}$ ou ceux des espèces dissoutes ${ }^{13} \mathrm{Cl}^{12} \mathrm{C}$ du gaz carbonique ou des bicarbonates, ${ }^{34} \mathrm{~S} /{ }^{32} \mathrm{~S}$ des sulfates ou des sulfures, ${ }^{15} \mathrm{~N} /{ }^{14} \mathrm{~N}$ des nitrates... Les abondances isotopiques permettent d'identifier l'origine des constituants et de reconstituer les phé-

\section{Contributions of isotopic techniques to the understanding of mineral water resources}

The first isotopes used were those of water itself, being an intrinsic part of the molecule and forming ideal tracers. Oxygen 18 and deuterium finally quashed the notion of juvenile water by demonstrating that mineral water had a meteoric origin. They also make it possible to locate the zone supplying the mineral water system by determining its mean depth on the basis of isotopic height gradients. Tritium from thermonuclear devices which was particularly abundant in precipitation during the decade 1963-1973 was used at an early stage to identify the presence of recent, post-nuclear water in mineral water springs.

Isotopes of dissolved elements, being exposed to chemical and biochemical fractionating, are a more delicate matter. They are used to determine the origin of the elements and to untangle the history of the water. Carbon 13 has been used to demonstrate the deep magmatic origin of the $\mathrm{CO}_{2}$ in most mineral waters that contain it. It is used to investigate the interactions between the $\mathrm{CO}_{2}$ gas, the bicarbonates and the limestone matrix, knowledge of which is essential for radiocarbon dating of ground water. The initial hopes that sulphur 34 would make it possible to distinguish sulphates dissolved in evaporites from those resulting from the oxidation of pyrite are now tempered by the extent and complexity of the fractionings resulting from the action of micro-organisms. Nitrogen 15 is still little used in the field of mineral waters, in which nitrate concentrations are usually very low. 
nomènes physiques, chimiques et biologiques ayant affecté la répartition isotopique (fractionnements).

Les isotopes radioactifs comme le tritium $\left({ }^{3} \mathrm{H}\right)$ de la molécule d'eau, le carbone $14\left({ }^{14} \mathrm{C}\right)$ ou le chlore 36 $\left({ }^{36} \mathrm{Cl}\right)$ se désintègrent régulièrement en fonction du temps. Ils peuvent permettre des mesures chronologiques ou "d'âge d'après modèle ». Il est nécessaire en effet de retrouver leur activité originelle et de vérifier qu'une production in situ ou une dilution par mélange ou par échange n'aient pas perturbé la seule décroissance radioactive.

Cette communication présente brièvement les méthodes couramment utilisées en faisant référence à quelques résultats significatifs obtenus sur les gisements d'eau minérale. Les méthodes peu utilisées ou encore du domaine de la recherche seront juste mentionnées.

\section{UTILISATION DES ISOTOPES DE LA MOLÉCULE D'EAU}

Il est impossible de concevoir un meilleur traceur de l'eau que les variétés isotopiques constituant sa molécule.

Par commodité, parce que les variations des teneurs en isotopes stables d'un élément donné ne s'élèvent qu'à quelques fractions de pour cent pour les phénomènes naturels, il est habituel d'exprimer les résultats par la fonction $\delta$ pour mille, en valeur relative par rapport à un standard international :

$$
\delta \%_{0}=\left(\frac{R \text { échantillon }}{R \text { standard }}-1\right) \times 1000
$$

où

$$
R=\frac{\text { abondance de l'isotope lourd }}{\text { abondance de l'isotope léger }} .
$$

Pour l'eau et donc pour l'oxygène $18\left({ }^{18} \mathrm{O}\right)$ et le deutérium $\left({ }^{2} \mathrm{H}\right)$ ce standard est le SMOW qui représente la composition moyenne des océans.

Le tritium $\left({ }^{3} \mathrm{H}\right)$ de période 12,43 ans est un émetteur bêta $\left(\beta^{-}\right)$. Les concentrations des eaux sont habituellement exprimées en Unités Tritium (U.T.). Une U.T. correspond à la présence d'un atome de tritium pour $10^{18}$ atomes d'hydrogène banal. Un litre d'eau dont la concentration est de 1 U.T. présente une radioactivité de 0,118 Becquerel (118 m Bq).

\subsection{Origine météorique de l'eau minérale}

En considérant leurs teneurs en oxygène 18 et deutérium on peut affirmer que toutes les eaux minérales françaises ont une origine météorique [1] :

- tout d'abord parce que les eaux minérales à l'émergence présentent des teneurs en ${ }^{18} \mathrm{O}$ et ${ }^{2} \mathrm{H}$ toujours assez proches à la fois de la composition des eaux météoriques locales et de celle des sources banales de leur environnement. Le fait que les teneurs isotopiques des sources minérales suivent la variabilité régionale des pluies est un argument déterminant.

- ensuite parce que toutes les eaux analysées se situent dans un diagramme $\delta{ }^{18} \mathrm{O}-\delta \mathrm{D}$, sur la droite des eaux météoriques définie par CRAIG [2] de pente sensiblement égale à 8 et d'ordonnée à l'origine ou excès en deutérium compris entre 10 et 14 .

Par exemple, sur la figure $I$ où ont été reportées les teneurs de sources thermominérales du Sud-Est de la France [3] les points représentatifs sont tous très proches de la droite météorique et ce, dans un domaine très large de variations depuis des valeurs très négatives $\left(-100 \delta \mathrm{D}\right.$ et $\left.-13,8 \delta{ }^{18} \mathrm{O}\right)$ pour des sources situées dans les Alpes comme Monetier ou la Liche des Chamois comportant des zones de recharge à l'altitude moyenne de $2300 \mathrm{~m}$ [4] jusqu'à des valeurs plus fortes de $-40 \delta \mathrm{D}$ et $-7 \delta^{18} \mathrm{O}$, à Jaujac en Ardèche avec zone d'alimentation à l'altitude de $450 \mathrm{~m} \mathrm{[5]}$.

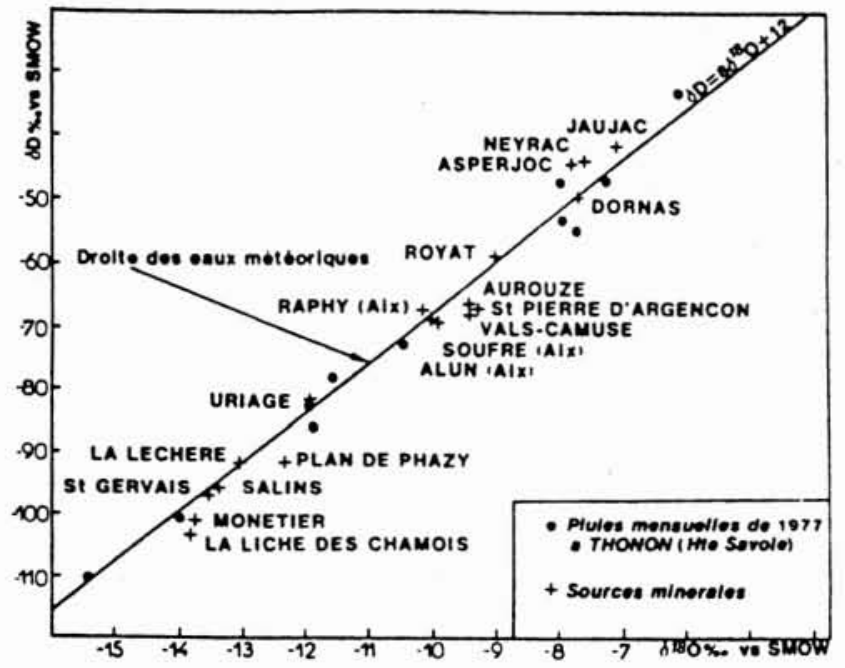

1. Variations des compositions isotopiques en oxygène 18 et en deutérium des sources minérales du Sud-Est de la France.

Si les eaux minérales étaient juvéniles, elles présenteraient des teneurs isotopiques sans doute homogènes et de toute façon très différentes de celles observées puisque l'on situe les concentrations de l'eau juvénile dans l'intervalle +5 à $+9 \delta \%$ en ${ }^{18} \mathrm{O}$ et -80 à $-40 \delta \%$ o en ${ }^{2} \mathrm{H}[6]$.

\subsection{Localisation de la zone d'alimentation}

Les teneurs isotopiques des pluies varient en fonction de l'altitude. C'est une conséquence de la thermodépendance qui voit, sous nos latitudes et à l'échelle des moyennes, les teneurs isotopiques des pluies dépendre de la température moyenne au sol [7]. Il en résulte la possibilité de déterminer l'altitude moyenne de l'aire d'alimentation d'une source minérale en situant sa teneur isotopique moyenne sur une droite régionale isotope stable-altitude. Cette droite est construite à partir des teneurs des pluies ou de préférence de sources aux bassins versants bien connus sélectionnées à différentes altitudes.

Une des premières applications de ce type [8-9] a été la localisation, sur le plateau de Gavot à $850 \mathrm{~m}$ d'altitude, de 
la zone d'alimentation des eaux minérales d'Evian à partir d'une étude fine de la variation des teneurs en ${ }^{18} \mathrm{O}$ des précipitations sur la rive sud du Léman. A partir des teneurs en ${ }^{18} \mathrm{O}$ des sources échantillonnées à différentes altitudes sur le massif du Sancy [10] (fig. 2), les altitudes respectives de $1500 \mathrm{~m}$ pour l'impluvium des sources minérales du Mont-Dore et de $1200 \mathrm{~m}$ pour celles de la Bourboule ont été déterminées, ce qui est compatible avec les structures géologiques de cette région volcanique.

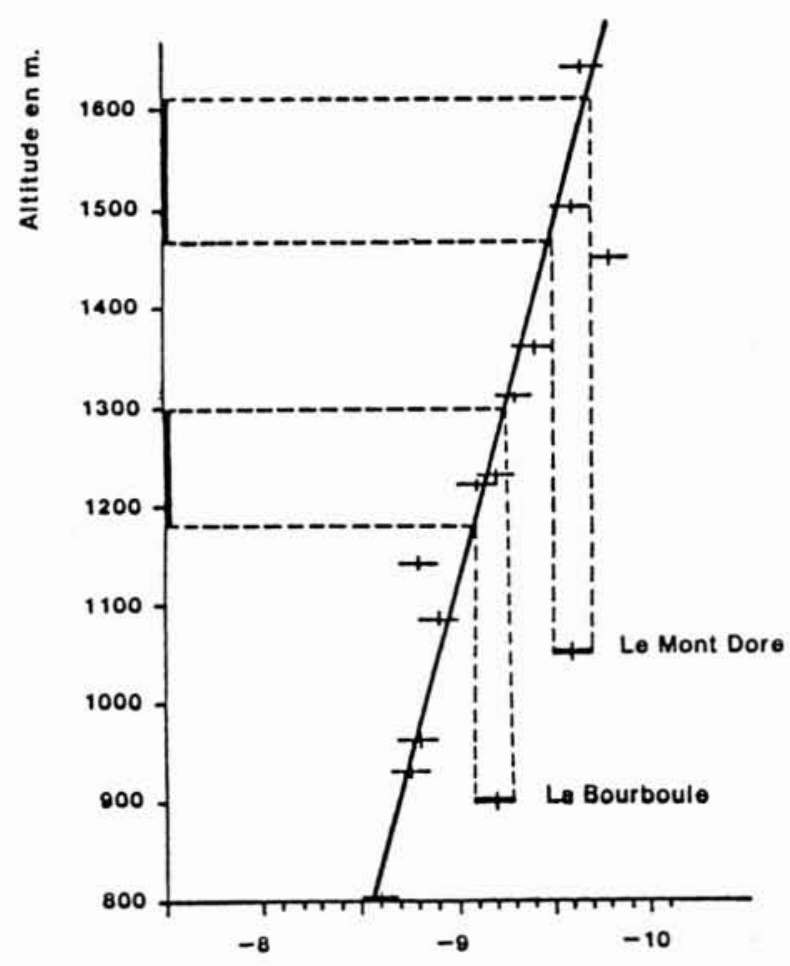

2. Relations entre la teneur en ${ }^{18} \mathrm{O}$ et l'altitude du bassin d'alimentation établie pour les sources du massif du Sancy.

Dans ce cas les gradients sont faibles, de l'ordre de $-0,16 \%$ en ${ }^{18} \mathrm{O}$ par $100 \mathrm{~m}$ d'élévation, valeur comparable à celle de $-0,15 \%$ trouvée entre 1200 et $2000 \mathrm{~m}$ dans les Pyrénées orientales [11] probablement parce qu'une grande partie de la recharge provient de la neige et que l'effet d'altitude et de température sur la neige est négligeable. Ailleurs ils sont généralement voisins de $0,22 \%$ o mais cette variabilité des gradients de 0,15 à $0,30 \%$ o impose d'établir au cas par cas la droite de variation locale si l'on veut une bonne précision sur l'altitude de la zone d'alimentation du circuit minéral.

\subsection{Le problème de l'effet paléoclimatique}

La localisation de la zone d'alimentation doit être abordée avec circonspection si l'on estime avoir affaire à des eaux très vieilles. Il est possible en effet que des eaux minérales infiltrées lors d'une période plus froide que l'actuelle comme le dernier glaciaire, enregistrent un appauvrissement de leurs teneurs en isotopes lourds dû à la baisse générale des températures, effet paléoclimatique qui se surajoute au seul effet de l'altitude.

Des études géochimiques, isotopiques et des datations menées sur des aquifères captifs des grands bassins sédimentaires français ont révélé la réalité de cet effet paléoclimatique. C'est ainsi que les sources minérales chaudes d'Eugénie-les-Bains et de Barbotan qui sont des exutoires de l'aquifère des sables sous-molassiques de l'Eocène du Bassin d'Aquitaine sont appauvries de $1,5 \delta{ }^{18} \mathrm{O}$ par rapport aux eaux actuelles qui s'infiltrent sur leur zone d'alimentation constituée par leur affleurement [12]. Il en est de même de l'eau du forage thermal de Nancy exploitée depuis l'aquifère des grès du Trias inférieur lorrain et appauvrie de $1,5 \delta{ }^{18} \mathrm{O}$ par rapport aux infiltrations actuelles sur leur affleurement dans le massif vosgien [13].

\subsection{Vérification des échanges avec l'encaissant}

On doit envisager la possibilité qu'ont les eaux météoriques infiltrées très profondément et portées à des températures élevées d'échanger l'oxygène 18 de la molécule d'eau avec celui des silicates ou carbonates constituant le réservoir. Mais dans ce cas, le point représentatif de l'eau minérale sur un diagramme ${ }^{18} \mathrm{O}-{ }^{2} \mathrm{H}$ ne se situe plus sur la droite des eaux météoriques mais en dessous puisqu'il y a eu enrichissement en ${ }^{18} \mathrm{O}$ au contact de la roche dont les teneurs sont fortes $(+5 \mathrm{a}+10 \%$ ) sans que les teneurs en ${ }^{2} \mathrm{H}$ n'en soient affectées, le réservoir étant pratiquement dépourvu en minéraux hydrogénés. Ce phénomène de déplacement a été observé sur les champs géothermiques [14] mais n'a pas été mis en évidence sur les sources minérales françaises à l'exception peut-être de la région de Corenles-Eaux dans le Cantal [15]. Nous pensons que cet échange n'est effectif qu'à partir d'une température profonde de $250^{\circ} \mathrm{C}$ dans le meilleur des cas, et dépend aussi de la nature de la roche encaissante, de son état d'altération et des paramètres hydrauliques commandant le temps de séjour de l'eau.

\subsection{Datation de l'eau minérale par le tritium}

Le tritium, isotope radioactif de l'hydrogène $\left({ }^{3} \mathrm{H}\right)$ est produit naturellement par la composante neutronique du rayonnement cosmique sur l'azote atmosphérique. La teneur induite dans les pluies est de l'ordre de 5 U.T. Mais la plus importante production de tritium est artificielle. Elle est née des premiers essais de la bombe « $\mathrm{H} »$ en 1952. Ces essais aériens ont provoqué dans la stratosphère des injections successives si bien que les teneurs des précipitations ont été multipliées par 1000 en 1963, sous nos latitudes [16]. Depuis l'été 1963, l'année du « pic majeur » les concentrations en tritium des pluies ont décru de façon exponentielle par suite de l'arrêt de la grande majorité des essais mais elles restent dans les années 90 encore 3 fois plus élevées (15 U.T.) qu'avant les premiers essais.

Au niveau de l'interprétation, cette production artificielle permet de différencier les eaux minérales qui résultent de précipitations sans ${ }^{3} \mathrm{H}$ thermonucléaire d'avant 1952 de celles résultant de précipitations formées dans une atmosphère contenant du ${ }^{3} \mathrm{H}$ nucléaire postérieurement à cette date. Le seuil de différenciation se situe actuellement à 2 U.T. qui est le seuil de mesure en routine du tritium 
même si le seuil véritable est encore plus bas puisque la teneur en ${ }^{3} \mathrm{H}$ des pluies avant 1952 étant de 5 U.T. il n'en subsiste 42 ans après qu'au plus 0,5 U.T. compte tenu de la décroissance radioactive. Les contrôles effectués sur de nombreuses eaux minérales françaises indiquent très souvent des eaux anciennes antérieures à 1952.

Pour les eaux minérales contenant du tritium il est difficile d'estimer l'âge de l'eau si l'on ne dispose pas d'une série d'analyses permettant de dégager une évolution des teneurs sur de nombreuses années et de choisir un modèle d'interprétation de type piston ou mélange. Par exemple l'eau minérale d'Evian ne contenait pas de tritium en 1963 [17]. La poursuite des mesures jusqu'à ce jour a permis d'observer un pic de tritium à la source Cachat en 1979, en réponse à l'impulsion majeure dans les précipitations de 1963. Le temps de filtration de l'eau est au moins égal à 16 ans (fig. 3) et le grand amortissement de l'impulsion initiale permet d'envisager un temps de séjour plus élevé de l'ordre de 50 ans [18].

A défaut de longues chroniques on peut en 1994 retenir la « recette » suivante :

— si la teneur de l'eau minérale dépasse 25 U.T. son âge moyen est de quelques dizaines d'années parce qu'elle est marquée par les fortes teneurs des pluies du pic de 1963 ; - si la teneur est comprise entre 10 et 25 U.T., il s'agit généralement d'une eau infiltrée durant la dernière décennie mais il peut aussi s'agir d'une eau post nucléaire se mélangeant pour partie avec une eau ancienne;

- si la teneur est comprise entre 2 et 10 U.T., il s'agit d'un apport d'eau post-nucléaire dans une eau ancienne.

\subsection{Mise en évidence des mélanges à l'émergence}

L'eau minérale peut difficilement rejoindre la surface sans être affectée par un mélange avec des eaux superficielles. Ainsi on observe généralement dans la zone d'émergence plusieurs sources correspondant à des mélanges en proportions différentes mais appartenant toutefois à la même lignée géochimique. Pour étudier ces mélanges généralement binaires, il est usuel d'examiner les relations de chaque élément de la minéralisation de tous les points d'eau avec l'élément conservatif le plus représentatif des eaux profondes comme les chlorures, le sodium ou les sulfates suivant les cas.

$\mathrm{Si}$ le contexte le permet, les isotopes de l'eau, puisque partie intégrante de la molécule, restent les meilleurs marqueurs de ce mélange. C'est ainsi qu'il est souvent possible de distinguer dans une aire d'émergences, la (ou les) source(s) principale(s) exempte(s) de tritium parce qu'ancienne, des sources secondaires affectées d'un mélange avec des eaux plus superficielles récentes et donc marquées par quelques unités Tritium, proportionnellement à l'importance du mélange. On a démontré également à l'aide des isotopes stables que l'eau minérale de Badoit (SaintGalmier) est la résultante d'un mélange entre une composante profonde gazeuse, très minéralisée et appauvrie en oxygène 18 et une composante du granite à moyenne profondeur qui se distingue cependant des aquifères de la plaine du Forez (fig. 4). Ce marquage résulte du fait que le circuit profond a une aire d'alimentation sur les monts du Forez à une altitude plus élevée que le circuit à moyenne profondeur sur les monts du Lyonnais et (ou) un temps de séjour très grand entraînant un effet paléoclimatique.

\section{III — UTILISATION DES ISOTOPES DES ES- PÈCES DISSOUTES}

\subsection{Origine du carbone minéral dissous}

Les rapports des isotopes stables ${ }^{13} \mathrm{C}^{12} \mathrm{C}$ sont exprimés en $\delta{ }^{13} \mathrm{C} \%$ par rapport à un étalon de référence (P.D.B.), une

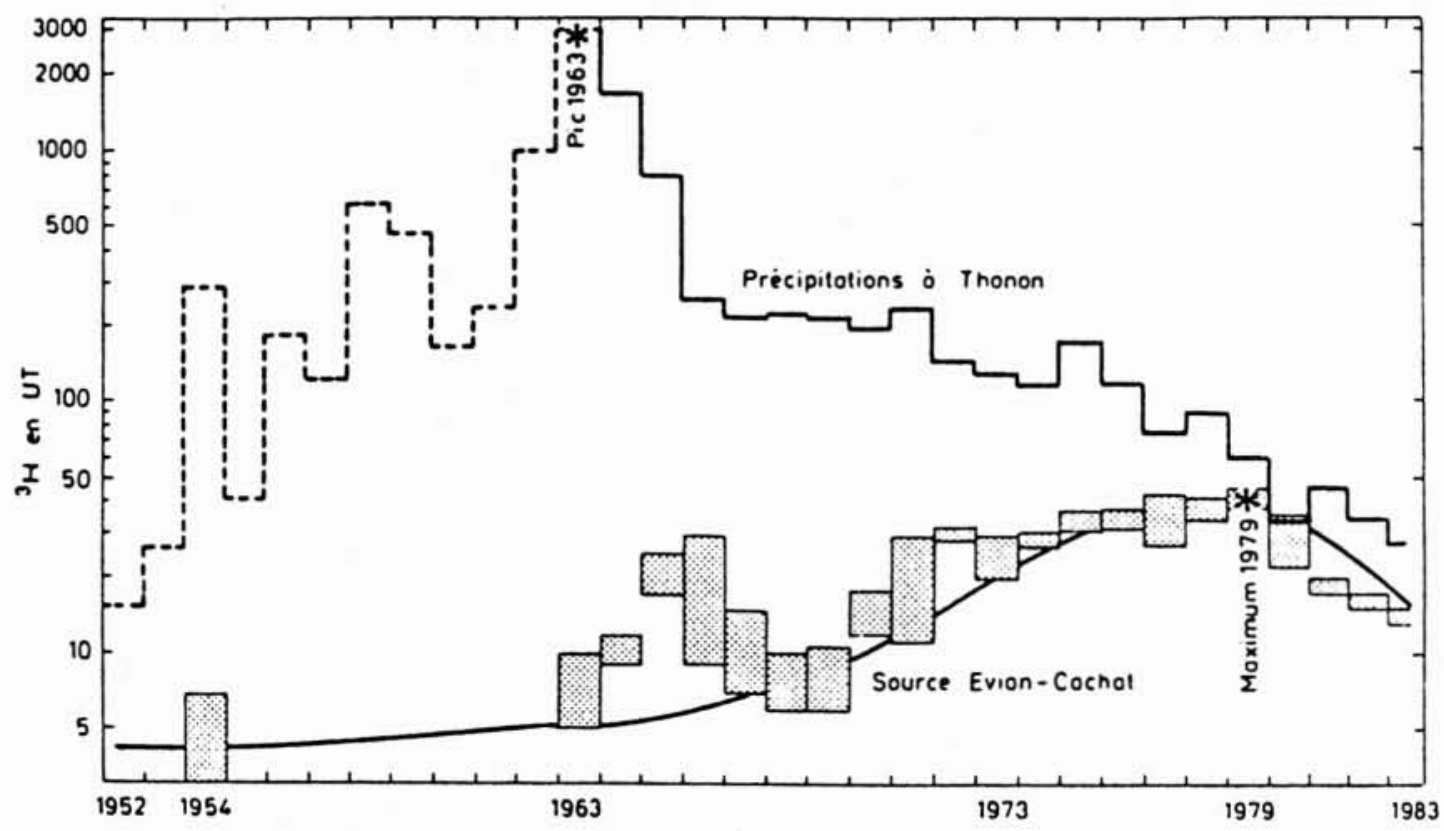

3. Evolution des teneurs en tritium dans les précipitations et la source minérale d'Evian-Cachat. 


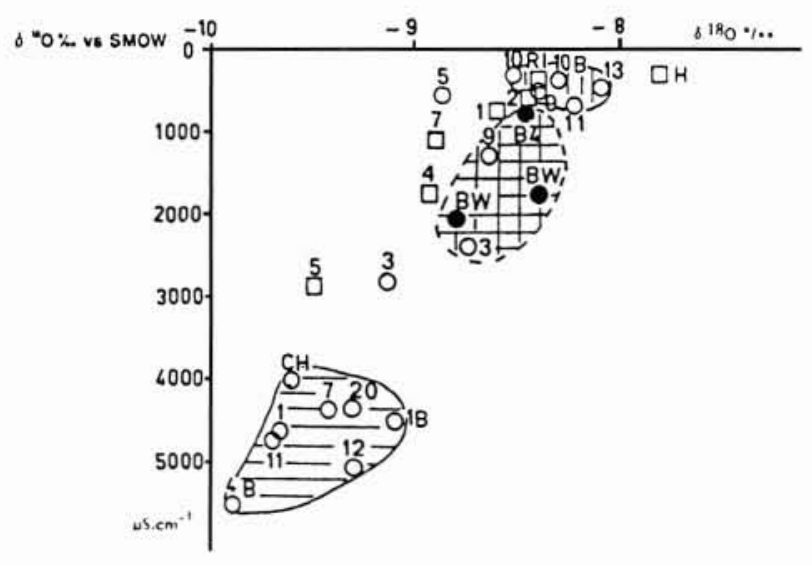

4. La source Badoit résulte du mélange entre une composante profonde très minéralisée et appauvrie en oxygène 18 et une composante du granite à moyenne profondeur.

bélemnite de la Pee Dee formation du Crétacé de Caroline du Sud. Les $\delta{ }^{13} \mathrm{C}$ du carbone minéral dissous, bicarbonates $\left(\mathrm{HCO}_{3}^{-}\right)$et gaz $\left(\mathrm{CO}_{2}\right)$ permettent quand ils sont employés avec précaution de retracer l'origine du carbone. Le problème est particulièrement d'acuité pour les sources minérales à dégagement gazeux.

La minéralisation carbonatée d'une eau souterraine s'acquiert communément par dissolution, d'une part du $\mathrm{CO}_{2}$ du sol résultant de la minéralisation par les micro-organismes de la matière organique du sol, d'autre part des carbonates du sol et des roches en contact avec l'eau. La matière organique a une composition isotopique de $-27 \pm 5 \%$ [19] et le $\mathrm{CO}_{2}$ biogénique légèrement enrichi, une valeur moyenne de $-24 \%$. La matrice calcaire des sédiments marins a une teneur isotopique moyenne de $0 \pm 2 \% \delta{ }^{13} \mathrm{C}[20]$. Si la mise en solution des carbonates s'effectue sans fractionnement, il n'en est pas de même de la mise en solution du $\mathrm{CO}_{2}$, toutefois peu fractionnante $\left(-1,1 \%\right.$ à $\left.25^{\circ} \mathrm{C}\right)$ et surtout de sa dissociation en bicarbonates qui enrichit les bicarbonates de $+8 \delta{ }^{13} \mathrm{C}$ par rapport au $\mathrm{CO}_{2}$ ambiant. Ainsi le $\delta{ }^{13} \mathrm{C}$ du carbone minéral dissous d'une eau souterraine dépend à l'infiltration en milieu ouvert des proportions de $\mathrm{CO}_{2}$ dissous et de bicarbonates donc de la $\mathrm{p} \mathrm{CO}_{2}$ et du $\mathrm{pH}$. Il évolue dans l'aquifère en milieu fermé en fonction de l'état de saturation de l'eau vis-à-vis des carbonates du réservoir. Les $\delta{ }^{13} \mathrm{C}$ du carbone minéral sont dans les conditions normales compris entre - 18 dans le sol et $-12 \%$ à saturation. La précipitation éventuelle de calcite appauvrit légèrement par effet de bilan le carbone minéral dissous car elle est enrichie d'environ $2 \%$.

Dans des conditions plus exceptionnelles qui peuvent être celles des circuits très profonds, la production de $\mathrm{CO}_{2}$ in situ est plus perturbatrice :

- La fermentation méthanique dans des milieux très réducteurs entraîne un fractionnement isotopique considérable de l'ordre de $50 \%$ [21] entre le $\mathrm{CH}_{4}$ appauvri (par exemple $\delta{ }^{13} \mathrm{C}=-50 \%$ et le $\mathrm{CO}_{2}$ enrichi $(0 \%$ ) transformés à parts égales de la matière organique $(-25 \%)$. L'eau rendue agressive par ce dégagement de $\mathrm{CO}_{2}$ retourne à saturation par dissolution de carbonate.
L'effet conjugué d'un apport de $\mathrm{CO}_{2}$ plus riche en ${ }^{13} \mathrm{C}$ et d'une nouvelle mise en solution de calcite enrichit le $\delta{ }^{13} \mathrm{C}$ du carbone minéral dissous. Des valeurs à $-5 \%$ sont couramment atteintes.

- L'arrivée par les grands accidents du socle de $\mathrm{CO}_{2}$ magmatique avec un $\delta{ }^{13} \mathrm{C}$ voisin de $-5 \%$ [22] provoque de la même façon un enrichissement en ${ }^{13} \mathrm{C}$ du carbone minéral dissous. Mais les conséquences en sont généralement plus spectaculaires car ces dégagements de $\mathrm{CO}_{2}$ extrêmement abondants arrivent à masquer complètement la minéralisation initiale de la zone d'infiltration en imposant leur marquage à l'eau minérale carbogazeuse compte tenu de la pression partielle de $\mathrm{CO}_{2}$ très élevée en profondeur.

Une application sur l'origine du carbone a été menée à partir d'un inventaire des analyses isotopiques effectuées sur le $\mathrm{CO}_{2}$ des gaz associés aux sources minérales françaises [3-15-23-24-25-26]. Leurs $\delta{ }^{13} \mathrm{C}$ sont rassemblés sur la figure 5 avec des indications sur leur provenance et la composition du dégagement gazeux.

- Pour les sources carbogazeuses typiques dont le $\mathrm{CO}_{2}$ constitue l'essentiel de la phase gazeuse en dépassant généralement $95 \%$, la composition isotopique du carbone 13 du $\mathrm{CO}_{2}$ libre est très regroupée autour de la valeur $-6 \pm 2 \%$ et est comparable à celle du carbone des gaz volcaniques directement libérés du magma. $\mathrm{Le} \mathrm{CO}_{2}$ a donc une origine endogène et résulte de phénomènes géologiques profonds comme le dégazage du manteau.

- Pour les sources à dégagements gazeux où prédomine l'azote en dépassant souvent $90 \%$, le $\mathrm{CO}_{2}$ a une composition isotopique qui varie de -12 à $-20 \delta{ }^{13} \mathrm{C} \%$ et indique une origine biogénique pour le carbone. Cette origine exogène est confirmée par les teneurs en azote et en argon assez voisines de celles de l'atmosphère. Ces gaz sont done véhiculés par des eaux de pluie infiltrées profondément qui remontent à la surface à la faveur de failles. - On n'a pas retrouvé en France de teneurs isotopiques du $\mathrm{CO}_{2}$ appartenant au domaine des carbonates marins et indiquant clairement la décomposition des calcaires à haute température (thermodécarbonatation).

Cette application porte sur le gaz $\mathrm{CO}_{2}$ se dégageant d'un griffon d'eau minérale ou sur la tête d'un forage. Il conviendrait en toute rigueur d'établir la teneur en ${ }^{13} \mathrm{C}$ du carbone minéral total dissous en profondeur, avant le point de bulle de l'eau carbogazeuse. En effet le départ de $\mathrm{CO}_{2}$ dissous appauvri par rapport aux bicarbonates entraîne par effet de bilan dans un système non réapprovisionné, un enrichissement du carbone minéral dissous restant. De telles vérifications sont en cours sur des forages d'exploitation profonds équipés pour de tels prélèvements.

\subsection{La datation de l'eau minérale par le carbone 14}

En l'absence de tritium, la datation par le radiocarbone peut être tentée. La période de demi-vie du carbone 14 est de 5730 ans et sa vie moyenne est donc de $5730 / \mathrm{Ln} 2$, soit 8267 ans. Si l'on suppose que la minéralisation carbonatée reste constante et que seule l'activité initiale en ${ }^{14} \mathrm{C}\left(A_{0}\right)$ décroît, la mesure de l'activité résiduelle $\left(A_{t}\right)$ en pourcents de carbone moderne $(\mathrm{pcm})$ permet de 


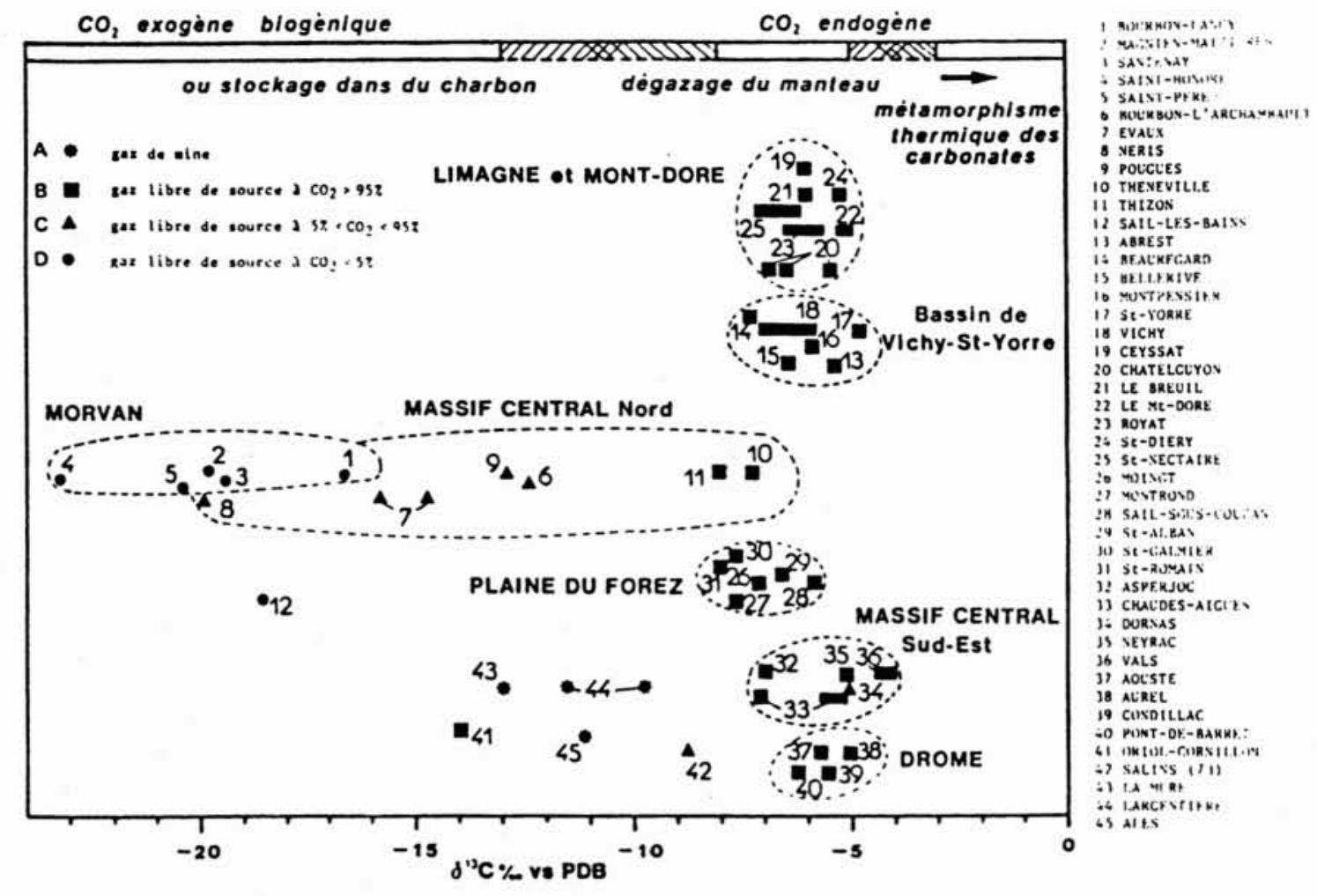

5. Teneurs en ${ }^{13} \mathrm{C}$ et origine du $\mathrm{CO}_{2}$ libre des sources minérales.

calculer le temps de séjour $\left(T_{S}\right)$ de l'eau minérale en admettant un écoulement souterrain de type piston :

$$
A_{t}=A_{0}{ }^{*} \exp -\left(T_{S} / 8267\right) \text { en pcm }
$$

et

$$
T_{S}=8267^{*} \operatorname{Ln}\left(A_{0} / A_{t}\right) \text { en années . }
$$

Toute la difficulté réside dans la détermination de l'activité initiale $\left(A_{0}\right)$. Suivant les réactions déjà évoquées pour le carbone 13, l'essentiel du carbone minéral dissous résulte de l'activité en ${ }^{14} \mathrm{C}$ du $\mathrm{CO}_{2}$ du sol, fixée à $100 \%$ de carbone moderne (avant les essais thermonucléaires) et de l'activité en ${ }^{14} \mathrm{C}$ nulle des carbonates «morts" du sol et du réservoir. La valeur attendue est donc de $50 \mathrm{pcm}$ mais l'expérience a montré que l'activité initiale était très supérieure. De très nombreux modèles ont donc été proposés pour évaluer $A_{0}$ soit à l'aide des teneurs isotopiques, soit en considérant les paramètres chimiques et isotopiques. Ils sont résumés dans deux articles de synthèse [27-28].

Quelques datations ont été obtenues sur les eaux minérales sulfurées sodiques [29], dans les Pyrénées à Cauterets (5 100 ans sur le groupe nord) et à Luchon (14 000 ans), en Corse à Guagno-les-Bains (6 000 ans) qui confirment les vitesses d'écoulement extrêmement lentes des eaux dans la profondeur des massifs de granite. En milieu sédimentaire les temps de séjour peuvent aussi être très élevés et ont été estimés par exemple pour les eaux minérales chaudes issues des sables sous-molassiques d'Aquitaine enregistrant un marquage paléoclimatique (cf. 2.3) à 29500 ans à Eugénie-les-Bains et 20000 ans à Barbotan. On doit hélas renoncer à la datation par le carbone 14 des eaux minérales carbogazeuses dont la minéralisation carbonatée est héritée en quasi-totalité d'un $\mathrm{CO}_{2}$ profond magmatique et de carbonates sédimentaires ou hydrothermaux à carbone 14 " mort ".

\subsection{Origine du soufre et activité bactérienne}

Les isotopes utilisés sont les isotopes stables les plus abondants de l'atome de soufre, ${ }^{32} \mathrm{~S}$ banal $(95,02 \%)$ et ${ }^{34} \mathrm{~S}(4,21 \%)$. Pour le rapport ${ }^{32} \mathrm{~S} /{ }^{34} \mathrm{~S}$ l'étalon de référence est la troílite $(\mathrm{FeS})$ de la météorite de Canyon Diablo aux Etats-Unis (CDT). L'étude isotopique du soufre peut renseigner sur l'origine des espèces dissoutes et rend possible l'identification des processus géochimiques et biochimiques qui les affectent. Quand il s'agit de sulfates, les études sont facilitées si l'on dispose aussi de la composition isotopique de l'oxygène $\left(\delta^{18} \mathrm{O}\right)$.

Au-delà des teneurs héritées des précipitations, les eaux continentales peuvent se minéraliser en sulfates en lessivant des formations géologiques contenant du gypse ou de l'anhydrite ou aussi en oxydant les sulfures solides comme la pyrite $\left(\mathrm{FeS}_{2}\right)$. La dissolution des minéraux sulfatés n'entraîne aucun effet isotopique et le sulfate dissous a donc les mêmes teneurs que le sulfate solide initial. Toutefois les teneurs en ${ }^{34} \mathrm{~S}$ des évaporites précipitées à partir des saumures marines ont varié au cours des temps géologiques de +10 à $+30 \%$ [30] si bien que l'on peut rechercher l'origine du sulfate de l'eau sur la courbe du marquage géologique du gypse, distinguer origine triasique et oligocène. L'oxydation du sulfure ne conduit pas à un fractionnement significatif en ${ }^{34} \mathrm{~S}$. Plus précisément, on ob- 
serve un léger appauvrissement pour le sulfate produit si la réaction est biochimique. Le fractionnement est quasiment nul lorsque le processus est chimique sauf à très haute température où il se produit un enrichissement. En première approximation on peut donc avancer que la teneur en ${ }^{34} \mathrm{~S}$ du sulfate formé par oxydation dépend de celle du sulfure. Les sulfures présents dans les roches signées ont des teneurs proches de celles de la troïlite servant de référence ( $\delta=0 \%$ par définition) mais les sulfures d'origine sédimentaire présentent des teneurs s'étalant sur une gamme de valeurs d'une amplitude au moins égale à $80 \%$ (de -40 à $+40 \%$ [31]. Cette variabilité des teneurs et son chevauchement avec la gamme des évaporites complique singulièrement la recherche de l'origine des sulfates d'une eau minérale quand on se trouve dans un contexte sédimentaire où l'ubiquité de l'origine se pose vraiment.

Grossièrement les résultats disponibles sur quelques eaux minérales françaises permettent de distinguer isotopiquement 2 groupes:

- celui des fortes valeurs, à sulfates dérivant des évaporites, dont les $\delta{ }^{34} \mathrm{~S}$ sont assez regroupés entre +15 et $+20 \%$ comme par exemple à Vittel $(+16 \pm 0,5 \%)$ [25] ou pour les sources chaudes du Briançonnais à l'Ouest du front de chevauchement $(+16 \pm 1 \%$ [ [32] ;

- celui des sulfates relativement appauvris dérivant de l'oxydation des sulfures métalliques dont les teneurs isotopiques sont habituellement inférieures à $+11 \%$, particulièrement pour les eaux ayant circulé dans les granitoïdes, par exemple dans les Vosges à Plombières $(+10,7+11,8 \%$ ) , dans les Pyrénées orientales à la Preste $(+6,5 \%$ ) [23] ou dans le Massif Central pour la source Badoit à Saint-Galmier $(+6,4 \%)$ et à Royat $(+11 \%)$ [33].

Cependant cette distinction n'est plus valable s'il s'agit d'une eau sulfurée dans laquelle sont présents sulfures et sulfates témoignant de phénomènes d'oxydo-réduction. En effet la réduction bactérienne du sulfate dissous enrichit le sulfate résiduel en isotopes lourds et les produits réduits sont appauvris. Le fractionnement instantané est alors important contrairement aux réactions précédentes et une valeur de 1,040 est communément admise dans le cas d'une réduction par les bactéries Désulfovibrio désulfuricans [31]. Le fractionnement apparent qui en résulte entre sulfates et sulfures est une fonction exponentielle de la fraction restante du sulfate réactant. Il témoigne en quelque sorte de l'ampleur de l'activité bactérienne. La réduction du sulfate est par ailleurs marquée sur un diagramme ${ }^{34} \mathrm{~S}-{ }^{18} \mathrm{O}$ par une droite de pente moyenne égale à 4 entre le sulfate initial et le sulfate résiduel [34].

Une étude isotopique du soufre dans les eaux thermominérales des Landes (BRGM, 1985) indique un fractionnement résultant entre sulfates enrichis et sulfures, voisin de $23 \%$, comparable à celui d'autres sources sulfurées françaises (Allevard, Enghien-les-Bains, Puzzichello). Ce fractionnement est plus faible que celui connu pour les eaux géothermales du Dogger du Bassin Parisien qui atteint jusqu'à $45 \%$ [35] dans un milieu réducteur sans doute plus confiné. Dans le cas des Landes, l'étude isotopique des formes du soufre permet d'apprécier l'ampleur de la réduction bactérienne à partir d'une minéralisation sulfatée acquise au contact des diapirs triasiques. Les fortes valeurs du $\delta^{34} \mathrm{~S}$ (de l'ordre de $+25 \%$ ) attestent de l'existence d'une réduction même quand les sulfures ne sont plus présents à l'émergence par suite d'une complète réoxydation comme à Dax.

\subsection{Autres isotopes pouvant être utilisés}

La géochimie isotopique de l'azote, ${ }^{15} \mathrm{~N} /{ }^{14} \mathrm{~N}\left(\delta^{15} \mathrm{~N}\right)$ des nitrates et de l'ammonium est maintenant bien connue [36], particulièrement dans les milieux superficiels où elle est très utile pour rechercher l'origine d'une pollution de l'eau ou étudier un phénomène de dénitrification. L'azote 15 est encore peu utilisé dans le domaine des eaux minérales dont les concentrations en nitrates sont généralement faibles, voire souvent nulles dans les circuits profonds. D'un point de vue fondamental, les signatures isotopiques de l'azote magmatique mériteraient d'être mieux connues.

Le rapport isotopique strontium 87 radiogénique (produit de la désintégration du rubidium) sur strontium 86 est caractéristique d'une roche donnée. L'eau du circuit hydrothermal qui l'altère va donc acquérir une marque d'origine. Cette utilisation initiée sur les champs géothermiques relève encore de la recherche scientifique.

Le chlore 36, isotope, radioactif, émetteur $\beta^{-}$, produit dans la stratosphère par activation de l'Argon 36 , a une période d'environ 300000 ans qui est bien adaptée à la datation des circuits hydrothermaux très profonds. La faible réactivité chimique de l'ion chlorure en solution en fait un excellent traceur dont l'usage est appelé à se développer dès que les difficultés analytiques auront été vaincues. En effet le seul dispositif capable de mesurer en routine des rapports de $10^{-13}$ à $10^{-15}$ entre l'isotope rare et les isotopes stables du même élément est la spectrométrie de masse par accélérateur de particules, machine qui n'est pas disponible en France.

\section{CONCLUSION}

Les grands principes du marquage isotopique naturel des eaux minérales ont été présentés.

A partir de quelques exemples, il a été montré que les méthodes isotopiques sont très performantes pour la connaissance initiale du système hydrominéral, dans la recherche de l'origine de l'eau, des gaz et des minéralisations comme dans la reconstitution des phénomènes chimiques ou biochimiques et des conditions hydrodynamiques qui ont marqué son trajet, particulièrement dans la partie terminale. Tous ces phénomènes contribuent à modeler un profil isotopique caractéristique d'une eau minérale autorisant aussi son utilisation pour l'authentification de la ressource comme pour le suivi régulier en cours d'exploitation de la qualité du fluide.

Les classifications présentées dans cette synthèse et bâties à partir des concentrations isotopiques constituent un premier guide pour l'interprétation des résultats mais ne doivent pas être considérées comme des recettes infaillibles. Le recours à toutes ces méthodes ne doit pas non plus être systématique. Avant leur mise en œuvre on recommandera à l'exploitant de consulter un spécialiste, seul à même de conseiller, après un examen du contexte hydrogéologique et hydrochimique, sur l'opportunité et les limites d'une méthode isotopique.

\section{Bibliographie}

[1] Blavoux B. et BerTHIER F. (1985), - Les originalités hydrogéologique et technologique des eaux minérales. Bull. Soc. géol. France (8), t. I, n 7, pp. 1033-1044. 
[2] CRAIGH H. (1961), - Isotopic variations in meteoric waters. Science, 133, pp. 1702-1703.

[3] Blavoux B., Dazy J. et Sarrot-Reynaud J. (1982). Information about the origin of thermomineral waters and gas by means of environmental isotopes in eastern iranian Azerbaïdjan and the southeast of France. Journal of $\mathrm{Hy}$ drology, 56, pp. 23-38.

[4] Poulain P.A. (1977). - Les eaux minérales et thermominérales dans le département des Hautes-Alpes. Thèse $3^{\mathrm{c}} \mathrm{cy}-$ cle, Univ. Grenoble I, Grenoble.

[5] JEANTIN M. (1979). - Les sources minérales carbogazeuses de la Haute-Ardèche. Etude géologique et hydrogéologique. Thèse $3^{\text {e }}$ cycle, Univ. Grenoble I, Grenoble.

[6] TAYLOR H.P. (1974). - The application of oxygen and hydrogen isotope studies to problem of hydrothermal alteration and ore deposition. Econ. Geol., 69, pp. 843-883.

[7] DansGaARD W. (1964). - Stable isotopes in precipitation. Tellus, 16, 4, pp. 436-468.

[8] Glangeaud L., Blavoux B., Fontes J.C., Letolle R., OLIVE Ph. (1967). - Utilisation des méthodes isotopiques dans l'étude des eaux minérales. La Presse Thermale et Climatique, $2^{\mathrm{e}}$ trimestre 1967, pp. 1-4, Imprimerie Gaignault, Issoudun.

[9] BLavoux B. (1969). - Les sources minérales d'Evian. La Houille Blanche, 1, pp. 21-29.

[10] JАСОВ P. (1975). - Contribution à l'étude géochimique des eaux froides et thermominérales du massif du Mont-Dore. Thèse $3^{\mathrm{e}}$ cycle, Univ. Paris VI, Paris.

[11] Krimissa M., Chery L., Foulllac C. (1993). - Isotopic study of thermomineral springs in the eastern Pyrénées: correlation with the origin of air masses and the altitude of recharge. Proc. on applications of isotope techniques in studying past and current environmental changes in the hydrosphere and the atmosphere, International Atomic Energy Agency, Vienna, I.A.E.A.-SM-329/74P.

[12] Blavoux B., Dray M., Fehri A., Olive Ph., Groning M., Sonntag C., Hauquin J.P., Pelissier G., Pouchan P. (1993). - Paleoclimatic and hydrodynamic approach to the Aquitaine basin deep aquifer (France) by means of environmental isotopes and noble gases. Proc. on applications of isotope techniques in studying past and current environmental changes in the hydrosphere and the atmosphere, International Atomic Energy Agency, Vienna, I.A.E.A.-SM329/60P.

[13] Blavoux B., Olive Ph. (1981), - Radiocarbon dating of groundwater of the aquifer confined in the lower triassic sandstones of the Lorraine region (France). Journal of $\mathrm{Hy}$ drology, 54 (1981), pp. 167-183.

[14] CRAIG H. (1963). - The isotopic geochemistry of water and carbon in geothermal areas. Nuclear geology of geothermal areas, Spoleto 1963, Tongiorgi (Ed.) C.N.R., lab. di Geologia Nucleare, Pisa, pp. 17-54.

[15] DEMONT J.M. (1981). - Etude isotopique de quelques circulations hydrothermales fossiles et actuelles. Thèse $3^{\mathrm{e}}$ cycle, géochimie, Univ. Paris VII, Paris.

[16] Olive Ph. (1970). - Contribution à l'étude géodynamique du cycle de l'eau dans l'hémisphère nord par la méthode du tritium. Thèse Doctorat d'Etat ès Sciences Naturelles, Paris, $138 \mathrm{p}$.

[17] Blavoux B., Glangeaud L., Levêque P., Olive Ph. (1964). - Hydrodynamique et teneur en tritium des eaux du bassin d'Evian (France). C.R. Ac. Sc. Paris, t. 259, pp. 4323-4326.

[18] Hubert P., Olive Ph., Molicova H. (1991), - Modélisation par une loi gamma de la distribution des temps de séjour de l'eau dans des systèmes hydrologiques en régime permanent. Rencontres hydrologiques Franco-Roumaines, Ecole des Mines de Paris, 3-6 septembre 1991.

[19] Troughton J.H. (1972). - Carbon isotope fractionation by plants. 8th Intern. Conf. on Radiocarbon dating, Lower Hutt, Nouvelle Zelande, 2, pp. 421-438.

[20] CRAIG H. (1953). - The geochemistry of the stable carbon isotopes. Geochim. Cosmochim. Acta 3, pp. 53-92.

[21] Balabane M., Galimov E., Hermann M., Letolle R. (1987). - Hydrogen and carbon isotope fractionation during experimental production of bacterial methane. Org. Geochim., 11, 2, pp. 115-119.

[22] Deines P., Gold D.P. (1973). - The isotopic composition of carbonatite and kimberlite carbonates and their bearing on the isotopic composition of deep-seated carbon. Geochim. Cosmochim. Acta, 37, pp. 1709-1733.

[23] Baubron J.C., Bosch B., Degranges P., Halfon J., Leleu J., Marcé A., Sarcia C. (1978). - Recherches géochimiques sur les eaux thermales d'Amélie-les-Bains (Pyrénées-orientales) et de Plombières-les-Bains (Vosges). Isotope Hydrology, II, proc. symp. Neuherberg, 1978. I.A.E.A., pp. 568-612.

[24] Mercier-Batard F, et Risler J.J. (1982), - Les gaz associés aux eaux thermominérales. Presse thermale et climatique, 119, 3, pp. 139-149.

[25] Annales des Mines (1983). - Fichier des sources d'eaux minérales françaises. $\mathrm{N}^{\circ} 8-9$.

[26] BLavoux B., Dazy J. (1990). - Caractérisation d'une province à $\mathrm{CO}_{2}$ dans le bassin du Sud-Est de la France. Hydrogéologie $n^{\circ} 4$, 1990, pp. 241-252.

[27] FONTES J.C., GARNIER J.M. (1979). - Determination of the initial ${ }^{14} \mathrm{C}$ activity of the total dissolved carbon: a review of the existing models and a new approach. Water Res. Res., 15,2 , pp. 399-413.

[28] MоoK W.G. (1980). - Carbon-14 in hydrogeological studies. Handbook of environmental isotope geochemistry. Fritz P., Fontes J.C., Eds, vol. 1, pp. 49-74, Elsevier.

[29] Terroirs et Thermalisme de France (1992). - Sous la direction de C. Pomerol et J. Ricour, Editions du BRGM.

[30] Claypool G.E., Holser W.T., Kaplan I.R., Sakal H., ZAK I. (1980). - The age curves of sulfure and oxygen isotopes in marine sulfate and their manual interpretation. Chem. Geology, 28, pp. 199-260.

[31] KROUSE H.R. (1980), - Sulfur isotopes in our environment. In: Handbook of Environmental Isotope geochemistry, P. Fritz and J.C. Fontes Eds., vol. 1, chap. 11, pp. 435-471, Elsevier.

[32] Bosch B., Dazy J., Lepiller M., Olive Ph., Poulain P., SARROT-REYNAUd J. (1976). - Données nouvelles sur quelques sources thermominérales des Alpes françaises. Proc. Int. Congress Thermal Waters, A.I.H.S., Athens, pp. 32-40.

[33] Batard F., Maisonneuve J., RisLer J.J. (1978). - La province hydrominérale des eaux carbogazeuses d'Auvergne. Rev. Sc. Nat. d'Auvergne, vol. 44, pp. 7-32.

[34] MiZUTANI Y., RAFTER T.S. (1969). - Isotopic composition of sulphate in rain water. Gracefield, New Zealand. N.Z. J. Sci., 12, pp. 69-80.

[35] Fouillac C., Fouillac A.M., Criaud A. (1989), - Etude isotopique du soufre des sulfures et des sulfates dans l'aquifêre géothermal du Dogger, Bassin parisien Hydrogéologie $\mathrm{n}^{\circ} 3,1989$, pp. 219-227.

[36] MARIOTTI A. (1986). - La dénitrification dans les eaux souterraines, principes et méthodes de son identification : une revue. Journal of Hydrology, 88 (1986), pp. 1-23. 